\title{
The Role of Xenobiotic Enzyme Genes of the First and Second Phases in the Pathogenesis of Fetal Growth Restriction Syndrome
}

\section{Mavlyanova NN*}

Republican Specialized Scientific and Practical Medical Center of Obstetrics and Gynecology Under the Ministry of Health of the Republic of Uzbekistan, Uzbekistan

*Corresponding Author: Mavlyanova NN, Republican Specialized Scientific and Practical Medical Center of Obstetrics and Gynecology Under the Ministry of Health of the Republic of Uzbekistan, Uzbekistan.
Received: June 16, 2020

Published: September 30, 2020

(C) All rights are reserved by Mavlyanova NN

\section{Abstract}

The article presents the results of molecular genetic studies of genes of biotransformation enzymes of xenobiotics of the first and second phase in pregnant women with fetal growth restriction syndrome. Using the del/del genotype variants of the GSTM1 and GSTT1 genes and G alleles of the GSTP1 gene, one can determine the prognosis of the risk of developing fetal loss syndrome, characterized by impaired detoxification during pregnancy.

Keywords: GSTM1; GSTT1; Fetal Growth Restriction Syndrome (FGRS)

\section{Introduction}

Fetal growth restriction syndrome (FGRS) is a clinical syndrome caused by morphological and functional changes in the placenta and penetrant by limit of the growth and development of the fetus, its hypoxia, that arise as a result of the combined reaction of the fetus and placenta to various disorders of the pregnant woman $[1-4,9,10,11,13,17]$.

However, they have not yet been sufficiently studied as genetic predisposition factors in fetal growth limit syndrome. According to the literature, these genes are a rather complex object of study due to a number of their specific features [5-7,11-14,19-22]. These are overlapping substrate specificity, inducibility, and participation in the metabolism of endogenous compounds. But it is precisely these features of XBE that make it possible to assume that they can be genetic markers at all stages of the development of the disease from its initiation to the outcome and, accordingly, will make it possible to identify a predisposition, help in the early diagnosis of the disease, knowing the patient's genotype, make a prognosis of the course of the disease, and choose the most suitable therapy.

\section{Aim of the Study}

The aim of our research was to study the detectability of allelic variants of gene polymorphism of xenobiotic biotransformation enzymes of pregnant women with fetal growth limit syndrome taking into account the age of the pregnant.

\section{Materials and Research Methods}

The object and subject of the study were pregnant women with fetal loss syndrome (FLS), DNA samples from patients and healthy donors, glutathione transferase genes GSTM1 (1p13.3), GSTT1 (22q11.2) and glutathione transferase gene GSTP1 (IIe 105 Val).

The study included 143 pregnant women aged 19 to 34 years, observed at the clinic of Republican Specialized Scientific and Practical Medical Center for Obstetrics and Gynecology, Ministry of Health of the Republic of Uzbekistan (RSSPMCOG MH RUz). All pregnant women underwent general clinical, laboratory and functional studies according to the standard for diagnostics and therapy (2015). Molecular genetic testing of biomaterials (DNA) was carried out on the basis of the Department of Molecular Medicine and Cellular Technology Research Institute of Hematology and 
Blood Transfusion under the Ministry of Health of the Republic of Uzbekistan. The object and subject of the study were DNA samples of pregnant and healthy donors, glutathione transferase genes of the first phase - GSTM1 (1p13.3), GSTT1 (22q11.2) and the second phase - GSTP1 (IIe $105 \mathrm{Val}$ ).

During genetic studies, the population control was used as a comparison group, which was represented by DNA samples ( $\mathrm{n}=$ 72) of conditionally healthy ones from the DNA bank of this department. DNA samples were isolated from peripheral blood lymphocytes in accordance with a modified methodology. The concentration and purity of the extracted DNA was evaluated by measuring the optical density of DNA-containing solutions at a wavelength of 260 and $280 \mathrm{~nm}$ against TE on a NanoDrop 2000 spectrophotometer (USA). Genotyping of GSTT1 and GSTM1 polymorphism was carried out by PCR on programmable thermal cyclers CG-1-96 Corbett Research (Australia) and 2720 Applied Biosystems (USA), using test systems of LLC Litekh (Russia), according to the manufacturer's instructions.

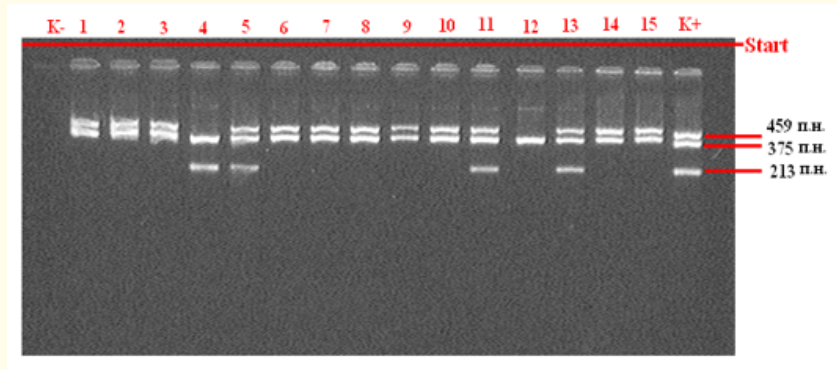

Picture 1: Statistical analysis of the results was carried out using the statistical software package "OpenEpi 2009, Version 2.3".

GSTM1 and GSTT1 gene detection electrophoregram.

(459 bps - GSTT1 gene, 375 bps - $\beta$-globin, 213 bps - GSTM1).
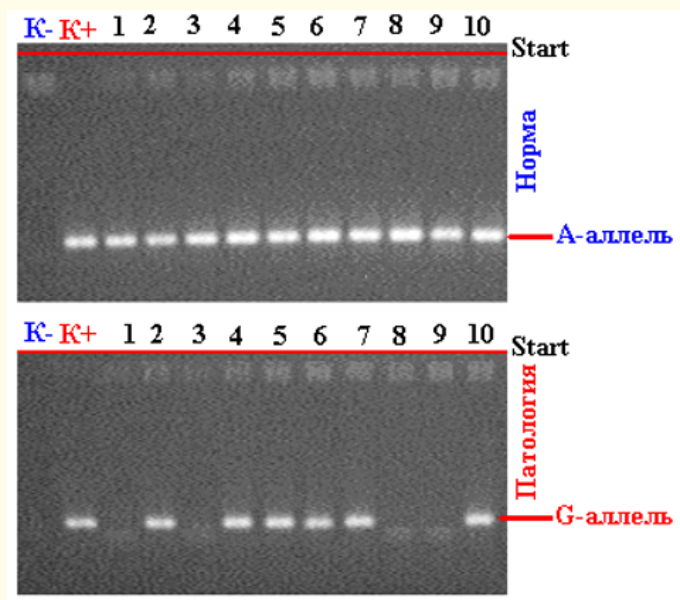

Picture 2: Electrophoregram for the detection of polymorphism $(\mathrm{A} / \mathrm{G})$ of the gene mutation -1 glutathione-S-transferase P1 (rs--).

$$
\begin{gathered}
\mathrm{K} \text { - Negative control } \\
\mathrm{K}+\text { Positive control } \\
1,3,8,9 \text { - Wild genotype A/A }
\end{gathered}
$$

2,4,5,6,7,10 - Heterozygous genotype A/G.

\section{Results and Discussion}

Clinical, laboratory and functional studies have shown that among the 143 pregnant women examined, fetal growth limit syndrome was detected in 105 , which amounted to $73.4 \%$. According to the severity degree, I - the degree of severity was diagnosed in 35 (33.3\%), II - the degree - in 48 (45.7\%) and III - degree - in 22, which amounted to $20.9 \%$, respectively.

Information on gene sequences and primer structure was obtained taking into account the original literary source [1] and Gene Bank. The characteristics of the genetic marker and the sequence of synthesized oligoprimers are shown in table 1,2.

Molecular genetic studies of the glutathione transferase genes GSTM1 (1p13.3), GSTT1 (22q11.2) and IIe 105 Val of the GSTP1 xenobiotic enzyme gene in the blood of pregnant women with FGLS revealed the following features of the distribution of alleles and genotypes of GSTM1 and GSTP1 gene polymorphisms (Table 3 and 4).

As it can be seen from table 3, in the main group of pregnant women with SPP, functional alleles of GSTM1 "+" were detected in $67.8 \%$ of cases (40), while deletion variants (non-functional) of GSTM1 (0/0) were detected in 32.2\% (19) cases. Whereas, functional allelic variants of GSTT1 “+” genotypes were detected in $20.3 \%$ of cases, and deletion variants in $79.6 \%$ (47) cases, respectively.

As the comparative analysis of the distribution frequencies of the alleles and genotypes of the IIe 105 Val polymorphism of the GSTP1 xenobiotic enzyme gene among 114 DNA samples in 57 pregnant women revealed the presence of the normal A allele and $64.1 \%$ of the $\mathrm{G}$ allele in $35.1 \%$ of cases. Whereas, in the control group, the frequency of occurrence of the mutant allele IIe $105 \mathrm{Val}$ of the GSTP1 xenobiotic enzyme gene was $12.5 \%$, which was 2.8 times lower in comparison to the main group $(\mathrm{P}<0.05)$.

For a detailed assessment of the prognostic criterion for the significance of the polymorphism of the genotypes of xenobiotic enzymes GSTM1, GSTT1 and GSTP1 in the development of fetal loss syndrome in pregnant women, we analyzed the results of analyzes depending on the presence of fetal loss syndrome (FGLS) and without.

The results of the study showed, that pregnant women with FGLS, combined functionally defective genotypes GSTM10/0 + GSTT10/0 were found in $28.2 \%$ of cases (11 pregnant women with FLS) than in the II control group individuals $(20.0 \%)$, which is 1.4 times higher than in this group.

In the group of pregnant women with FGLS "functionally unfavorable" A/G genotypes of the GSTP1 gene was found in $63.04 \%$ (29) versus $27.3 \%$ (3) of pregnant women without FLS, which was 2.3 times higher than the indicators of this groups $(\mathrm{P}<0.05)$. It should be noted that unfavorable homozygous genotypes were detected only in the I - group of pregnant women with FLS, which amounted to $17.4 \%$. 
The Role of Xenobiotic Enzyme Genes of the First and Second Phases in the Pathogenesis of Fetal Growth Restriction Syndrome

\begin{tabular}{|c|c|c|c|}
\hline No & Gene, localization & Polymorphism & The structure of oligoprimers \\
\hline \multirow{2}{*}{1} & \multirow{2}{*}{ GSTM1 (1p13.3) } & DELETION & $\begin{array}{r}\text { F 5-'GAACTCCCTGAAAAGCTAAAGC-3' } \\
\text { R 5'-GTTGGGCTCAAATATAGGGTGG -3' }\end{array}$ \\
\hline \multirow{2}{*}{2} & GSTT1 (22q11.2) & DELETION & $\begin{array}{r}\text { F 5'-TTCCTTACTGGTCCTCACATCTC-3' } \\
\text { R 5'-TCACCGGATCATGGCCAGCA-3' }\end{array}$ \\
\hline
\end{tabular}

Table 1: The sequence of oligonucleotide primers used for PCR.

\begin{tabular}{|c|c|c|c|}
\hline No & Gene, localization & Polymorphism & The structure of oligoprimers \\
\hline 1 & GSTP1 (11 (11.g13)) & Detection & $\begin{array}{c}\text { 5'-ACCAGGGCTCTATGGCCAA- } \\
\text { 5'-TGACCCGAGAAGAACGGGT-3', }\end{array}$ \\
\hline
\end{tabular}

Table 2

\begin{tabular}{|c|c|c|c|c|c|c|c|c|c|}
\hline \multirow{3}{*}{ No } & \multirow{3}{*}{ Groups } & \multicolumn{8}{|c|}{ Genotype Distribution Frequency } \\
\hline & & \multicolumn{2}{|c|}{ GSTM1 «+» } & \multicolumn{2}{|c|}{ GSTM1 (0/0) } & \multicolumn{2}{|c|}{ GSTT1 «+» } & \multicolumn{2}{|c|}{ GSTT1 (0/0) } \\
\hline & & $* \mathbf{n}$ & $\%$ & $* \mathbf{n}$ & $\%$ & $* \mathbf{n}$ & $\%$ & $* \mathbf{n}$ & $\%$ \\
\hline 1. & The main group, $\mathrm{n}=59$ & 40 & 67,8 & 19 & 32.2 & 12 & 20,3 & 47 & 79.6 \\
\hline 2 & The control group, $\mathrm{n}=72$ & 46 & 64.0 & 26 & 36.1 & 54 & 75.0 & 18 & 25.5 \\
\hline
\end{tabular}

Table 3: Distribution frequency of alleles and genotypes of del/del genes polymorphism of the GSTM1 and GSTT1 in pregnant and control groups.

$\mathrm{n}$ - Number of patients examined.

$* \mathrm{n}$ is the number of alleles studied.

\begin{tabular}{|c|c|c|c|c|c|c|c|c|c|c|c|}
\hline \multirow{3}{*}{ No } & \multirow{3}{*}{ Group } & \multicolumn{4}{|c|}{ Allele frequency } & \multicolumn{6}{|c|}{ Genotype distribution frequency } \\
\hline & & \multicolumn{2}{|c|}{ A } & \multicolumn{2}{|c|}{ G } & \multicolumn{2}{|c|}{$\mathbf{A} \backslash \mathbf{A}$} & \multicolumn{2}{|c|}{$\mathbf{A} / \mathbf{G}$} & \multicolumn{2}{|c|}{$\mathbf{G} / \mathbf{G}$} \\
\hline & & $\mathbf{n}$ & $\%$ & $\mathbf{n}$ & $\%$ & $\mathbf{n}$ & $\%$ & $\mathbf{n}$ & $\%$ & $\mathbf{n}$ & $\%$ \\
\hline 1. & The main group $n=57$ & 74 & 64.9 & 40 & 35.1 & 21 & 36,8 & 32 & 56,1 & 4 & 7,02 \\
\hline 2 & The control group $n=72$ & 126 & 87.5 & 18 & 12.5 & 57 & 79.2 & 14 & 19.4 & 1 & 1.4 \\
\hline
\end{tabular}

Table 4: The distribution frequency of alleles and genotypes of polymorphism IIe 105 Val of the GSTP1 gene in groups of patients and control.

$\mathrm{n}$ - Number of patients examined.

* $\mathrm{n}$ is the number of alleles studied.

\begin{tabular}{|c|c|c|c|c|c|c|}
\hline No & Genetic marker & SE & SP & AUC & OR (95\%CI) & ${ }^{*} \mathbf{p}$ \\
\hline 1 & del/del genes GSTM1 & 0.32 & 0.64 & 0.48 & $0.8 ; 0.4-1.74$ & 0.6 \\
\hline A & del/del genes GSTT1 & 0.80 & 0.75 & 0.77 & $11.7 ; 5.132-26.9$ & $<0.05$ \\
\hline B & GSTM1+GSTT1 & 0.86 & 0.43 & 0.65 & $7.8 ; 2.146-28.65$ & 0.0004 \\
\hline
\end{tabular}

Table 5: Predictive efficacy of the studied genetic markers. 
As it follows from table 5 , the indicators of the level of specificity and sensitivity of the del/+ polymorphism of the GSTT1 gene were $\mathrm{SE}=0.8$ and $\mathrm{SP}=0.75$, respectively, at significantly high values $(\mathrm{OR}=11.7 ; 95 \%$ CI 5.132-26.9). At the same time, the calculated AUC indicator demonstrates a high level of effectiveness for predicting the development of the disease, which indicates the possible independent effect of this polymorphism on the risk of pathology development.

The SE and SP indices of the combined variant of the del/+ polymorphisms of the GSTM1 + GSTT1 genes deviate towards sensitiv- ity and are equal to 0.86 and 0.43 , respectively, and the efficiency rating is 0.65 . These indicators also show a rather significant level of prognostic value of combinations of unfavorable genotypes as a genetic marker for predicting the development of fetal loss syndrome.

Then, studies of the expected and observed heterozygous frequencies of the IIe 105 Val polymorphism of the GSTP1 gene in pregnant women with FLS and without revealed distinctive features (Table 6).

\begin{tabular}{|l|c|c|c|}
\hline Groups & $\begin{array}{c}\text { Observed heterozygosity } \\
\left(\mathbf{H}_{\text {obs }} \mathbf{)}\right.\end{array}$ & $\begin{array}{c}\text { Expected heterozygosity } \\
\left(\mathbf{H}_{\mathbf{e x p}}\right)\end{array}$ & $\mathbf{D}^{*}$ \\
\hline Pregnant with FLS & 63,04 & 48,09 & $+0,31$ \\
\hline Pregnant women without FLS & 19,44 & 23,55 & -0.17 \\
\hline
\end{tabular}

Table 6: The difference between the expected and observed frequencies of heterozygosity of the IIe 105 Val polymorphism of the GSTP1 gene.

As it follows from the table, the observed, i.e. the actual distribution of GSTP1 gene A/G combinations is significantly higher than expected (63.04 versus 48.09 , respectively). The relative deviation $\mathrm{D}$ of the observed heterozygosity from that expected in the main group of patients was positive, i.e., $\mathrm{D}=+0.31$ ). Whereas in the group of pregnant women without FGLS, the distribution of A/G combinations of the GSTP1 gene in the expected group turned out to be higher (23.55 versus 19.44 , respectively). The deviation D of the observed heterozygosity from the expected one turned out to be negative, i.e. $\mathrm{D}=-0.17$.

Pregnant women with FGLS, the frequency of the observed heterozygosity of the Iie 105 Val polymorphism of the GSTP1 gene was $63.04 \%$, which was 3.2 times higher than that of pregnant women without FGLS, and the frequency of the expected heterozygosity was $48.09 \%$, which was 2.4 times higher indicators of pregnant women without FGLS $(\mathrm{P}<0.05)$.

An analysis of the results shows that the distribution of all genotypes of the IIe 105 Val polymorphism of the GSTP1 gene in the group of pregnant women and the control corresponds to PXB, indicating the absence of the influence of systematic or random factors that can change the genetic structure of populations. A study of the genetic structure of this marker revealed a relatively high level of expected heterozygosity in the main group of patients in relation to the control group ( $63.04 \%$ and $19.4 \%$, respectively.). In both groups, the indicator $\mathrm{D}$ is to the left of 0 , that means it is negative $(D<0)$. The revealed fact indicates higher frequencies of the expected heterozygotes, and not actually calculated heterozygotes.

Thus, an analysis of the association of intergenic combinations of zero polymorphisms of the GSTM1 and GSTT1 genes revealed that in the group of pregnant women with fetal loss syndrome, combinations of the homozygous del/del genotype responsible for a lower level of protein product synthesis are significantly more common. The chance of developing pathology in the presence of this combination of the genotypic variant of del/del genes GSTM1 and GSTT1 significantly increases: up to 7.8 times more compared to other genotypes $\left(\chi^{2}=12.4 ; \mathrm{P}=0.0004 ; \mathrm{OR}=7.8 ; 95 \%\right.$ CI 2.146 28.65). Whereas, the functionally unfavorable $G$ allele of the GSTP1 gene 2.7 times statistically significantly prevailed in the studied chromosomes of pregnant women with FLS compared with pregnant women without FLS $\left(\chi^{2}=4.6\right.$; $\mathrm{P}=0.03$; OR $=4.5$; 95\% CI1.06119.5).

Analysis of the results of molecular genetic studies shows that female individuals of the Uzbek population with combined zero genotypes of the xenobiotic enzymes GSTM1 and GSTT1, as well as hetero/homozygous genotypes of the IIe 105 Val GSTP1 polymorphism, have a tendency to risk fetal loss syndrome $\left(\chi^{2}=12.4 ; \mathrm{P}=\right.$ 0.0004; OR = 7.8; 95\% CI 2.146-28.65). 
Thus, the combined null genotypes GSTM10/0 + GSTT10/0 of the xenobiotic enzyme genes GSTM1 and GSTT1, as well as hetero (G/A)/homozygous $(G / G)$ genotypes of the IIe 105 Val polymorphism of the GSTP1 gene, are significant markers of an increased risk of loss syndrome fetus in Uzbekistan $(\mathrm{P}<0.05)$. Allele A and the functionally favorable genotype A/A IIe 105 Val of the GSTP1 gene are significant protective markers for the development of pathology $\left(\chi^{2}=18.6 ; \mathrm{P}<0.05 ; \mathrm{OR}=3.9 ; 95 \%\right.$ CI 2.023-7.07).

The results obtained indicate that the variants of polymorphisms of the GSTM10/0 + GSTT10/0 genotypes of the GSTM1 and GSTT1 genes, as well as the G/A IIe 105 Val genotypes of the GSTP1 gene, are significant prognostic criteria for the risk of fetal growth limit syndrome, which are caused by disorders of the detoxification process in the body in women during pregnancy.

\section{Conclusion}

- An analysis of the association of intergenic combinations of zero polymorphisms of the GSTM1 and GSTT1 genes revealed that in the main group of patients, combinations of the homozygous del/del genotype responsible for a lower level of protein product synthesis are significantly more common. The chance of developing pathology in the presence of this combination of the genotypic variant of del/del genes GSTM1 and GSTT1 significantly increases: up to 7.8 times more compared to other genotypes.

- Thus, the G allele and hetero/homozygous genotypes of the IIe 105 Val polymorphism of the GSTP1 gene are significant markers of an increased risk of developing fetal loss syndrome in Uzbekistan ( $\mathrm{P}<0.05$ ). Allele A and the functionally favorable A/A genotype are significant protective markers for the development of pathology $\left(\chi^{2}=18.6\right.$; $\mathrm{P}<0.05$; $\mathrm{OR}=$ 3.9; 95\% CI 2.023-7.07).

- $\quad$ Based on the variants of the del/del genotypes of the GSTM1 and GSTT1 genes and G alleles of the GSTP1 gene, one can determine the prognosis of the risk of developing fetal loss syndrome, characterized by a violation of the detoxification process of the body during pregnancy.

\section{Bibliography}

1. NA Davydova., et al. "Analysis of polymorphic variants of the T1, M1 and P1 glutathione S-transferase genes in patients with prostate cancer". XI Russian Oncological Congress: Congressional materials (2007): 224.

2. Ahmed-zade VA. "Pregnancy and childbirth with antiphospholipid syndrome: course, perinatal outcomes". Medical News 5 (2011): 81-85.
3. Barinova IV. "Pathogenesis of antenatal death: phenotypes of fetal loss and thanatogenesis". Russian Bulletin of the Obstetrician-Gynecologist 1 (2015): 68-76.

4. Bespalova ON. "Genetics of miscarriage". Journal of Obstetrics and Women's Diseases (2007): 81-95.

5. Gavalov SM., et al. "The association of gene polymorphism of xenobiotic biotransformation and detoxification enzymes with children with bronchial asthma features". Allergology 3 (2000): 14-21.

6. Guzov II. "Plasmogen activator inhibitor gene polymorphism 1 (PAI-1) - risk of developing obstetric pathology". www.cironline.ru/board/index.

7. Eliseeva Yu E. "Angiotensin-converting enzyme, its physiological role". Questions of Medical Chemistry 1 (2001): 15-21.

8. Lyubchich NI and Boboev KT. "Study of the role of coagulation system gene polymorphism in the occurrence of preterm birth in women of the Uzbek population". Medical Genetics 14.14 (2015): 37-41.

9. Makatsaria AD. "Antiphospholipid syndrome - immune thrombophilia in obstetrics and gynecology". (2007): 55-112.

10. Melkozerova OA., et al. "Molecular genetic predictors of abnormal uterine bleeding in girls born with intrauterine growth retardation syndrome". Obstetrics and Gynecology 11 (2016): 95-100.

11. Morozova KV and Lutsenko NN. "The role of gene polymorphism of antioxidant system enzymes in the genesis of miscarriage". Obstetrics, Gynecology and Reproduction 2 (2015): 54-61.

12. Tyszkiewicz OB. Features of the hemostatic system in women with habitual miscarriage and the presence of antiphospholipid antibodies in the blood plasma: author. dis. ... cand. medical science. - SPb (2004): 24.

13. Fedorova IA., et al. "Molecular genetic aspects of metanephrogenesis in norm and pathology: scientific publication". Russian Bulletin of Perinatology and Pediatrics 57.5 (2012): 45-51.

14. Khodzhaeva DA., et al. "The role of SOD2 gene polymorphism in women in the genesis of non-developing pregnancy". Bulletin of the Russian State Medical University 2 (2011): 134-136.

15. Amirchaghmaghi E., et al. "Gene expression analysis of VEGF and its receptors and assessment of its serum level in unexplained recurrent spontaneous abortion". Cell Journal 4.16 (2016): 538-545. 
16. Liu SY., et al. "Association between single nucleotide polymorphisms of 5'-untranslated region of GPx4 gene and male infertility". 28.3 (2011): 270-274.

17. Bermano G., et al. "Evidence that a polymorphism within the 3'UTR of glutathione peroxidase 4 is functional and is associated with susceptibility to colorectal cancer. School of Life Sciences, The Robert Gordon University, Aberdeen, AB25 1HG, UK". Genes Nutrition 2.2 (2007): 225-232.

18. Lin JC., et al. "Glutathione peroxidase 3 gene polymorphisms and risk of differentiated thyroid cancer". Surgery 145.5 (2009): 508-513.

19. Mlakar SJ., et al. "Antioxidant enzymes GSR, SOD1, SOD2, and CAT gene variants and bone mineral density values in postmenopausal women: a genetic association analysis". Menopause 19.3 (2012): 368-376.

20. Quick SK., et al. "Effect modification by catalase genotype suggests a role for oxidative stress in the association of hormone replacement therapy with postmenopausal breast cancer risk". Cancer Epidemiology, Biomarkers and Prevention 17.5 (2008): 1082-1087.

21. Seamus J Murphy., et al. "A population - based association study of SNPs of GSTP1, MnSOD, GPX2 and Barrett, s esophagus and esophageal adenocarcinoma". Carcinogenesis 28.6 (2007): 1323-1328.

22. Yang H., et al. "The association of GSTM1 deletion polymorphism with lung cancer risk in Chinese population: evidence from an updated meta-analysis". Science Report 5 (2015): 9392.

\section{Assets from publication with us}

- Prompt Acknowledgement after receiving the article

- Thorough Double blinded peer review

- Rapid Publication

- Issue of Publication Certificate

- High visibility of your Published work

Website: https://www.actascientific.com/

Submit Article: https://www.actascientific.com/submission.php

Email us: editor@actascientific.com

Contact us: +919182824667

Citation: Mavlyanova NN. "The Role of Xenobiotic Enzyme Genes of the First and Second Phases in the Pathogenesis of Fetal Growth Restriction Syndrome". Acta Scientific Microbiology 3.10 (2020): 84-89. 